\title{
O que tem sido divulgado em periódicos científicos online no Brasil sobre violência contra crianças e adolescentes
}

\author{
Daise Laís Machado Ferreira*, Zaida Aurora Sperli Geraldes Soler, D.Sc.**
}

*Enfermeira com atuação em pediatria, mestranda em Ciências da Saúde, foco temático Saúde da Criança e do Adolescente, pós-graduanda em Violência contra Crianças, Docente da Graduação da FAMERP, **Obstetriz, Enfermeira, Doutora e Livre Docente em Enfermagem Obstétrica, Docente e Orientadora da Graduaçâo e da Pós-graduação da FAMERP, Diretora Adjunta de Extensão de Serviços à Comunidade da FAMERP, Coordenadora do Programa de Enfermagem - Mestrado Acadêmico da FAMERP

\section{Resumo}

Introdução: A violência contra crianças e adolescentes é um grave problema de saúde pública em todo o mundo. Objetivo: $\mathrm{O}$ objetivo deste estudo foi apresentar uma revisão da bibliografia sobre violência infanto-juvenil. Método: Documentos bibliográficos nacionais veiculados online na íntegra em periódicos indexados na Biblioteca Virtual de Saúde (BVS) da rede Bireme. Resultados: Foram resenhados 83 artigos, veiculados online na íntegra de 1994 a 2012, das áreas de Saúde Coletiva, Psicologia, Medicina e Enfermagem. Conclusão: São necessárias mais pesquisas que subsidiem a prevenção, controle, notificação e respeito a questôes éticas, legais e humanísticas no contexto de violência infanto-juvenil no Brasil.

Palavras-chave: violência, violência doméstica, criança, adolescente.

\section{Abstract}

What has been published in scientific journals online in Brazil on violence against children and adolescent

Introduction: Child and adolescent abuse has been recognized as a major public health problem around the world. Objective: The objective of this study was to present a literature review of studies on child and adolescent abuse. Methods: National bibliographic full-text documents published in journals indexed by the Virtual Health Library (VHL) of Bireme's network. Results: 83 articles emphasizing sexual abuse and published in full from 1994 through 2012 have been reviewed, in the areas of Public Health, Psychology, Medicine and Nursing. Conclusion: Further researches on ethical, legal and humanistic issues are needed to subsidize prevention, control, and reporting of child and adolescent physical abuse in Brazil.

Key-words: violence, domestic violence, child, adolescent. 


\section{Resumen}

\section{Lo que se ha publicado en revistas científicas online en Brasil sobre la violencia contra los niños y adolescentes}

Introducción: Se señala la violencia contra los niños y adolescentes como un grave problema de salud pública mundial. Objetivo: El objetivo de este estudio fue presentar una revisión de la literatura sobre los malos tratos físicos contra niños y adolescentes. Métodos: Documentos bibliográficos nacionales difundidos íntegramente en periódicos indexados en la Biblioteca Virtual de Salud (BVS) de la red Bireme. Resultados: Se revisaron 83 artículos difundidos online en su totalidad entre 1994 y 2012, en las áreas de Salud Pública, Psicología, Medicina y Enfermería. Conclusión: Se necesitan más investigaciones sobre los contextos éticos, jurídicos y humanistas, para subsidiar la prevención, el control y la notificación de la violencia contra los niños y adolescentes en Brasil.

Palabras-clave: violencia, violencia doméstica, nińo, adolescente.

\section{Introdução}

A compreensão de violência é de uso de força ou poder, contra a própria pessoa, alguém ou algum grupo social vulnerável, com intenção de maltratar, provocando danos físicos, psicológicos e ou emocionais [1-3]. É um fenômeno frequente e crescente em todo o mundo e considerado como um grave problema de saúde pública, por sua dinâmica complexa que envolve aspectos físicos, psicológicos, sociais, éticos, legais e humanísticos. Pela sua complexidade e as consequências que acarreta exige que diferentes instituiçóes e políticas públicas promovam intervençóes coordenadas [1,3-8].

A violência doméstica inclui maus tratos que vão desde negligência até abuso físico, emocional e sexual. Quando está voltada à criança é ainda mais repudiada, por atingir seres que não podem se defender, por serem intrafamiliares, perpetradas por pessoas que deviam prestar cuidados, não castigos ou maus tratos e por ser uma importante causa de morte ou de morbidade entre crianças, que têm seu desenvolvimento físico, mental e social prejudicados, muitas vezes de forma irreversível [1-3,6-15].

Os profissionais de saúde têm papel importante na suspeita, percepçáo e notificação da violência doméstica contra crianças e adolescentes. Também devem conhecer dados epidemiológicos, fatores de risco, sinais de alerta, aspectos legais e as políticas públicas de saúde associadas e as consequências dos maus-tratos, para permitir o adequado processo de assistência e auxílio às vítimas [1-5].

Considerando lacunas existentes nas pesquisas, nas políticas públicas e nas ações institucionais de atenção a crianças e adolescentes vitimizados no
Brasil, nas últimas décadas, alguns grupos sociais e pesquisadores vêm lutando em defesa dos brasileiros menores. Há que se destacar que a partir de 1990 entrou em vigor o Estatuto da Criança e do Adolescente (ECA), Lei no 8.069, tendo como objetivo assegurar os direitos das crianças e dos adolescentes. Nele se destaca o papel dos profissionais de saúde e de educaçáo, que têm a responsabilidade e a obrigação de ao suspeitar ou identificar casos de violência, fazer a correta notificação. Também no ECA sáo explicitadas as penalidades para aqueles que são agressores ou que agem de forma omissiva ou comissiva quanto aos maus tratos na infância e adolescência $[2,8,15]$.

O cumprimento das determinações previstas no ECA exige a formação de Conselho Tutelar, um tipo de associação brasileira de proteção a crianças e jovens, com finalidade de tomar medidas que incluem orientaçôes e apoio às vítimas, a busca de abrigo, a comunicaçáo dos fatos à Vara da Infância e da Juventude [12] e a realizaçáo de encaminhamentos para os serviços de proteção social, jurídica ou de saúde mental [2,5-15].

A relevância na realização de estudos bibliográficos, conduzidos de forma sistematizada, é também permitir que se apresente um panorama da temática e da magnitude da problemática pesquisada. Desta forma podem se revelar as açóes que devem ser realizadas para prevenção de ocorrências, melhor atendimento aos afetados e para aperfeiçoar dispositivos legais, políticas de saúde e programas de educação, atenção, controle e de reabilitação, nos âmbitos físico e mental. Assim, este estudo tem como objetivo analisar periódicos científicos nacionais indexados em bases de dados de maior impacto acadêmico, di- 
vulgados de forma online, com abordagem explícita de violência contra crianças e adolescentes.

\section{Métodologia}

Trata-se de estudo bibliográfico sistematizado, com uso da internet e da integração com a Biblioteca Virtual em Saúde (BVS), por meio da Bireme (Centro Latino-Americano e do Caribe de Informação em Ciências da Saúde), nas Bases Eletrônicas Lilacs e SciELO Brasil e pelo endereço eletrônico $h t t p: / /$ scholar.google.com.br, utilizando-se como descritores "violência doméstica, violência contra crianças e adolescentes e maus tratos".

Para a coleta dos dados elaborou-se um instrumento com a finalidade de extrair os elementos necessários para a elaboração da resenha de cada artigo, tomando-se todo cuidado no sentido de respeitar as ideias explicitadas pelos autores em suas pesquisas.

Vale ressaltar que a pesquisa bibliográfica visa descrever as diferentes nuances e aspectos de um problema que mereça ser estudado de forma aprofundada, por meio de referencias teóricas publicadas em documentos, com revisão ou atualização da literatura relacionada. Podem ser usadas tanto descriçóes quantitativas quanto qualitativas, dependendo dos objetivos do estudo e das variáveis que se deseja estudar. As informaçóes bibliográficas científicas são organizadas, classificadas e indexadas em diferentes bancos de dados, com utilização da Internet, em redes de informação interligadas a bibliotecas nacionais e internacionais.

A seleção, classificação e análise das publicaçóes tomou por base a coerência e pertinência dos documentos para sua seleçáo e ser divulgado na íntegra, sendo os resultados apresentados em Tabelas e na forma descritiva.

\section{Resultados}

Foram analisados 83 artigos científicos, publicados em 29 periódicos com divulgação online, texto completo gratuito livre acesso - base de dados Scielo, com publicaçóes entre 1994 a 2012. A apresentação dos resultados foi feita com base no agrupamento em quatro áreas temáticas/profissionais, segundo o tipo de publicação que era recomendada nos periódicos, como segue: Saúde Coletiva; Psicologia; Medicina e Enfermagem. São descritos os periódicos segundo cada área temática e buscou-se na homepage da CAPES, a qualificação Qualis Capes atual de cada periódico.

Observa-se na Tabela I que foram classificados neste estudo 83 artigos, publicados em 29 periódicos, a maioria, 43 (51,8\%), abordava temática da área de saúde coletiva, enquanto os restantes eram de revistas de psicologia (14 artigos), de enfermagem (19 artigos) e de medicina (7 artigos).

Tabela I - Área temática e número de periódicos e de artigos sobre violência contra crianças e adolescentes.

\begin{tabular}{lcc}
\hline \multicolumn{1}{c}{ Área Temática } & Periódicos & Artigos \\
\hline Saúde Coletiva & 9 & 43 \\
Psicologia & 7 & 14 \\
Enfermagem & 7 & 19 \\
Medicina & 6 & 7 \\
TOTAL & 29 & 83 \\
\hline
\end{tabular}

São apresentados a seguir, de forma descritiva, os periódicos e número de artigos, nas quatro áreas temáticas definidas neste estudo:

\section{Área Temática: Saúde Coletiva - 9 Periódicos e 43 Artigos}

- Ciência \& Saúde Coletiva - (15 artigos) - revista de edição mensal pela Associação Brasileira de Saúde Coletiva/Abrasco, com divulgação qualitativa ou quantitativa, de temas de saúde pública, Qualis/ Capes B1.

- Cadernos de Saúde Pública - (15 Artigos) - publicação mensal da Fundação Osvaldo Cruz- Rio de Janeiro, considerada a revista científica brasileira com maior fator de impacto na área da saúde coletiva, com Qualis/ Capes A1.

- Saúde e Sociedade - (5 Artigos) - periódico científico trimestral, com parceria editorial entre a Faculdade de Saúde Pública da USP (FSP) e da Associação Paulista de Saúde Pública (APSP). Qualis /Capes B2.

- Revista Saúde Pública - (3 artigos) - revista bimestral, de responsabilidade da Faculdade de Saúde Pública - USP - Sáo Paulo. A partir de 2012 passou a cobrar dos autores taxa de publicação. Qualis/Capes A2.

- Revista Brasileira de Saúde Materno Infantil- (2 artigos ) - revista trimestral, do Instituto Materno Infantil de Pernambuco (IMIP), Qualis/Capes B1.

- Physis Revista de Saúde Coletiva (1 Artigo) - publicação trimestral do Instituto de Medicina Social da UERJ. Conta com o apoio do Conselho 
Nacional de Desenvolvimento Científico e Tecnológico (www.cnpq.br) e do Convênio PROAP/ CAPES (Programa e Apoio à Pós-graduação da Coordenação de Aperfeiçoamento de Pessoal de Nível Superior). www.capes.gov.br. Qualis Capes B1.

- Interface - Comunicação, Saúde, Educação (1 artigo): publicação trimestral editada pela UNESP de Botucatu. Dá ênfase a pesquisas qualitativas, articulando comunicação e educação. Qualis/ Capes é A2 - Educação; B1 - Saúde Coletiva.

- Revista Brasileira de Epidemiologia - (1 artigo): publicação trimestral, pela Associação Brasileira de Pós-Graduação em Saúde Coletiva - ABRASCO. Qualis/Capes B1.

A seguir são mostrados os artigos e periódicos na área temática de Psicologia. A classificação Qualis/Capes foi anotada pelo extrato de avaliação de periódicos 2010 da Psicologia - Webequalis (SICAPES).

\section{Área Temática Psicologia - 7 periódicos -14 artigos}

- Psicologia Ciência e Profissão - (6 artigos) - publicação trimestral dos Conselhos Federal e Regionais de Psicologia. Qualis/Capes A2 - Psicologia.

- Estudos de Psicologia - (2 Artigos) - revista trimestral do programa de Pós-Graduação em Psicologia do Centro de Ciências da Vida da Pontifícia Universidade Católica de Campinas - PUC. Qualis/ Capes A2 - Psicologia e B1 na Saúde Coletiva.

- Aletheia - (1 artigo) - revista quadrimestral do Curso de Psicologia da Universidade Luterana do Brasil. Qualis/Capes B1 - Psicologia e B4 na Saúde Coletiva.

- Psico-USF - (1 artigo) - revista quadrimestral do Programa de Pós Graduação Stricto Sensu de Psicologia da Universidade São Francisco. Qualis/ Capes B1 - Psicologia e B3 Saúde Coletiva.

- Psicologia em Estudo - Maringá - (2 artigos) - revista trimestral publicada, pelo Departamento de Psicologia da Universidade Estadual de Maringá, Paraná. (www.dpi.uem.br). Qualis Capes A2;

- Psicologia: Teoria e Pesquisa - (1 artigo) - revista trimestral do Instituto de Psicologia da Universidade de Brasília - UNB, Qualis/ Capes A2 Psicologia e B3 interdisciplinar;

- Psicologia \& Sociedade - (1 artigo) - revista quadrimestral da Associaçáo Brasileira de Psicologia
Social - ABRAPSO, visa o desenvolvimento da Psicologia Social e interdisciplinar. Qualis/ Capes A2 - Psicologia.

Outra categorização dos artigos sobre violência contra crianças e adolescentes foi na área temática da Medicina, como se mostra a seguir.

\section{Área Temática Medicina - 6 periódicos -7 artigos}

- Jornal de Pediatria - (2 artigos) - publicação científica bimensal da Sociedade Brasileira de Pediatria (SBP), com a versão integral e de livre acesso nos formatos impresso e online, disponível em português e inglês no site http://www.jped. com.br, Qualis Capes B1.

- Revista da Associação Médica Brasileira - RAMB (1 artigo) - periódico científico bimestral, Qualis/ Capes B3.

- Revista Brasileira Psiquiatria - (1 artigo) - publicação científica trimestral da Associação Brasileira de Psiquiatria (ABP). Além dos números regulares publica dois suplementos anuais. Qualis/Capes B1.

- Revista Paulista de Pediatria - (1 artigo) - publicação científica trimestral nas áreas de saúde e doença do recém-nascido, lactente, criança e adolescente. Qualis/ Capes B2 - interdisciplinar.

- Revista Brasileira de Educação Médica - (1 artigo) - publicação trimestral sobre temas considerados relevantes nas áreas de Educação Médica. É, interdisciplinar e o Qualis Capes é B2 .

- Revista de Psiquiatria Clínica (1 artigo) - divulgação técnico-científica trimestral do Departamento e Instituto de Psiquiatria da Faculdade de Medicina da Universidade de São Paulo. Qualis Capes - B1 - interdisciplinar.

Por fim apresenta-se a área temática de Enfermagem, na classificação de artigos sobre violência contra crianças e adolescentes publicados em periódicos nacionais, com divulgação online. A classificação Qualis-Capes na área da Enfermagem foi a disponibilizada no Sistema Integrado Capes WEBQUALIS da CAPES.

\section{Área Temática - Enfermagem - 19 artigos em 7 periódicos}

- Revista Latino Americana de Enfermagem - (6 artigos) - órgão oficial de divulgação bimestral da 
Escola de Enfermagem da USP de Ribeirão Preto e do Centro Colaborador da OPS/OMS para o Desenvolvimento da Pesquisa em Enfermagem. Interdisciplinar, Qualis Capes - A1- Enfermagem.

- Revista Brasileira de Enfermagem - (5 Artigos) - (REBEn), criada em 1932, é o mais antigo periódico da enfermagem brasileira e órgão oficial de publicação bimestral da Associação Brasileira de Enfermagem (ABEN). Qualis/Capes - A2.

- Acta Paulista de Enfermagem - (3 Artigos) - revista da Escola Paulista de Enfermagem - Diretoria de Enfermagem - UNIFESP, periodicidade trimestral nas áreas de Ciências da Saúde. Qualis/ Capes A2.

- Revista Eletrônica de Enfermagem - (2 artigos) - Disponível no site http://www.fen.ufg.br/revista, é um periódico de acesso aberto, gratuito e trimestral, Qualis/Capes - B1 - Enfermagem e Interdisciplinar.
- Texto \& Contexto Enferm - (1 artigo) - Revista trimestral do Programa de Pós-Graduação em Enfermagem da Universidade Federal de Santa Catarina, filiada à Associação Brasileira de Editores Científicos -ABEC .Qualis/Capes A2 - Enfermagem.

- Cogitare Enfermagem - (1 artigo) - publicação trimestral do Departamento de Enfermagem da Universidade Federal do Paraná - UFPR, Qualis/ Capes - B4.

- Ciência, Cuidado e Saúde - Maringá- (1 Artigo) - publicação trimestral do Departamento de Enfermagem e Programa de Pós-Graduação em Enfermagem da Universidade Estadual de Maringá-UEM. Qualis /Capes - B1.

Na Tabela II estão os dados sobre ano de publicação dos artigos sobre violência contra crianças e adolescentes, segundo área temática dos periódicos. Verifica-se que a maior parte das publicaçóes ocorreu entre 2008 e 2012 (50-60,2\%).

Tabela II - Publicações sobre violência contra crianças segundo ano e área temática de publicação dos artigos.

\begin{tabular}{|c|c|c|c|c|c|}
\hline \multirow{4}{*}{ Ano de publicação } & \multicolumn{5}{|c|}{ Área temática de publicação } \\
\hline & \multirow{3}{*}{$\begin{array}{c}\text { Saúde Coletiva } \\
\qquad \mathrm{N}^{\circ}\end{array}$} & \multirow{3}{*}{$\begin{array}{c}\text { Psicologia } \\
\mathrm{N}^{\circ} \\
\end{array}$} & \multirow{3}{*}{$\begin{array}{c}\begin{array}{c}\text { Medicina/ } \\
\text { Enfermagem }\end{array} \\
\mathrm{N}^{\circ}\end{array}$} & \multicolumn{2}{|c|}{ Total } \\
\hline & & & & & \\
\hline & & & & $\mathrm{N}^{\circ}$ & $\%$ \\
\hline 1994 & & & & 1 & 1,2 \\
\hline 1998 & 2 & & & 2 & 2,4 \\
\hline 1999 & 5 & & 1 & 6 & 7,3 \\
\hline 2000 & 2 & & & 2 & 2,4 \\
\hline 2001 & - & 1 & 1 & 2 & 2,4 \\
\hline 2002 & 2 & 1 & 1 & 4 & 4,8 \\
\hline 2004 & 2 & & & 2 & 2,4 \\
\hline 2005 & 1 & 1 & 2 & 4 & 4,8 \\
\hline 2006 & 1 & 2 & 3 & 6 & 7,3 \\
\hline 2007 & 3 & & 1 & 4 & 4,8 \\
\hline 2008 & 4 & 2 & 2 & 8 & 9,6 \\
\hline 2009 & 4 & 1 & 6 & 11 & 13,2 \\
\hline 2010 & 4 & 3 & 1 & 8 & 9,6 \\
\hline 2011 & 10 & 1 & 3 & 14 & 16,9 \\
\hline 2012 & 2 & 2 & 5 & 9 & 10,8 \\
\hline TOTAL & 43 & 14 & 26 & 83 & 100 \\
\hline
\end{tabular}

\section{Discussão}

Este estudo constitui-se numa análise de artigos online científicos sobre violência contra crianças e adolescentes, de autores brasileiros. A cada ano, a produção de conhecimento e a comunicação científica na área da saúde crescem em todo o mundo, com aumento dos pesquisadores cadastrados, de número de publicaçóes e de qualidade dos periódicos. No Brasil isso também tem acontecido, com desigualdades entre as diferentes regiōes, predominando as redes de pesquisadores no eixo norte-sul do país [16].

Nesta revisão da literatura, os estudiosos sobre o assunto de violência infanto-juvenil são unânimes em destacar a magnitude deste problema e sobre a 
necessidade desta realidade ser reconhecida e conhecida em suas diferentes nuances. Com isso é possível obter subsídios para fazer diagnósticos abrangentes acerca desta problemática social e elaborar, propor e implementar estratégias de intervenção e de prevenção de sua ocorrência e dos agravos que dela resultam. No Brasil, a violência contra crianças e adolescentes é um problema relevante e ainda muito pouco pesquisado de forma aprofundada, para ser possível compreender tal fenômeno sobre os vários prismas associados e permitir açóes de prevençáo e intervenção adequadas [1-15,17-36].

Para análise das publicações nacionais sobre violência contra crianças e adolescentes fez-se a categorização em 4 áreas temáticas (Saúde Coletiva, Psicologia, Medicina e Enfermagem). O objeto de análise foi a qualidade dos periódicos, segundo as bases de dados de indexaçáo, denominando-as pelo Qualis, no Brasil, número de artigos e periódicos, ano de publicaçáo e foco temático, em cada uma das quatro áreas analisadas.

O Qualis foi desenvolvido pela CAPES para fundamentação do processo de avaliação da pós-graduação stricto sensu nacional, de acordo com o fator de impacto das revistas publicados no JCR -2002 (Journal of Citation Reports), para qualificá-las como internacional nível A, B ou C. No Brasil, os órgãos de pesquisa utilizam o Qualis para julgar projetos, estimulando a publicação em periódicos mais valorizados e relevantes em cada área.

Considerou-se as indexaçôes de cada periódico e de ser disponibilizado online, em texto completo, gratuito, na base de dados SciELO (Scientific Electronic Library Online): biblioteca eletrônica abrangendo selecionada coleção de periódicos científicos latino-americanos em todas as áreas do conhecimento, desenvolvida como parceria entre a FAPESP e a Bireme e apoio do CNPq. Analisando os periódicos incluídos neste estudo, nas quatro áreas profissionais categorizadas, observou-se maior número de publicaçóes com credenciamento para Qualis A1 ou A2- 3 (33,3\%) dos 9 periódicos da Saúde Coletiva; 6 ( $85,7 \%)$ dos 7 periódicos da Psicologia , quando analisado só a área e 2(28,6\%) quando visto no SICAPES; nenhum dos 6 periódicos da Medicina e $4(57,1 \%)$ dos 7 periódicos da Enfermagem, analisados na área.

Após sistemática análise foram classificados 29 periódicos e 83 artigos. Considerando a área temática teve-se na Saúde Coletiva, 9 (31\%) dos periódicos e $43(51,8 \%)$ dos artigos; na Psicologia 7
(24,1\%) dos periódicos e 14 (16,9\%) dos artigos; na Medicina, 6 (20,7\%) dos periódicos e 7 (8,4\%) dos artigos; e na Enfermagem 7 (24,1\%) dos periódicos e $19(22,9 \%)$ dos artigos.

Quanto aos anos de divulgação, dos 83 artigos teve-se apenas $4(4,8 \%)$ na década de $90 ; 11$ $(13,2 \%)$ entre os anos 2000 e 2005 e $38(45,8 \%)$ de 2006 a 2012. Analisando por área temática, foram publicados entre 2006 e 2012: na Saúde Coletiva, dos 43 artigos, 30 (69,8\%); na Psicologia, dos 11 (78,6\%); na Medicina, 6 (85,7\%) dos 7 artigos e na Enfermagem 15 (78,9\%) dos 19 artigos.

Categorizou-se os focos de investigação nos aspectos abaixo, tendo-se a frequência absoluta e percentual de cada área temática e a fundamentação da literatura, como segue:

- Epidemiológicos e psicossociais: 44 (53\%) dos 83 artigos, publicados nos periódicos das 4 áreas temáticas, correspondendo a $24(55,8 \%)$ da Saúde Coletiva; 5(35,7\%) da Psicologia; $4(57,1 \%)$ da Medicina e $11(57,9 \%)$ da Enfermagem, abordando esta temática as referências bibliográficas [1,2,5,6,12-14,18,19,22,24-26,33]

- Prevenção, conhecimento e notificação: 20 (24\%) dos 83 artigos, correspondendo a $11(25,6 \%)$ da Saúde Coletiva; 4 (28,6\%) da Psicologia; 1 $(14,3 \%)$ da Medicina e $4(21,1 \%)$ da Enfermagem. Das referências bibliográficas usadas neste estudo, deram ênfase a este aspecto as [4, $10,11,15,21,28]$

- Programas de intervenção profissional: 14 (16,9\%), sendo 7 (16,3\%) da Saúde Coletiva; 3 (21,4\%) da Psicologia; 1 (14,3\%) da Medicina e $3(15,85)$ da Enfermagem. Abordaram com mais especificidade a este aspecto as seguintes bibliografias citadas neste estudo [3,8,23,27,29,30,32,34-36]

- Implicaçóes éticas e legais: 5 (6,2\%), correspondendo a 1 (2,3\%) da Saúde Coletiva; 2 (14,3\%) da Psicologia; 1 (14,3\%) da Medicina e 1 (5,3\%) da Enfermagem, com as seguintes publicaçôes citadas neste estudo $[7,9,17,20,31]$

Ainda, vale destacar que a temática da violência sexual contra crianças e adolescentes tem merecido a atenção de muitos pesquisadores brasileiros, que ressaltam a iniquidade desta situação, o fator de risco para psicopatias, as questóes psicossociais e forenses, a problemática da notificação, a necessidade de reconhecimento e notificação e o desfecho judicial $[2,4,6,8,18-20,22,25-29,36]$. 


\section{Conclusão}

Os dados obtidos neste estudo bibliográfico mostram que são importantes para aferição do conhecimento produzido em diferentes enfoques e para delimitar as áreas profissionais e as variáveis que merecem maior amplitude de investigação, no que se refere à violência contra crianças e adolescentes no Brasil. Ainda, podem favorecer melhor orientação do profissional de saúde quanto às formas de prevenção, atuação, notificação e controle de violências contra crianças e adolescente.

\section{Referências}

1. Branco MAO, Tomanik EA. Violência doméstica contra crianças e adolescentes: prevençáo e enfrentamento. Psicologia \& Sociedade 2012;24(2):402-11.

2. Souto RQ, Lucena B, Paiva AA, Cavalcanti AL. Violência sexual em crianças e adolescentes: uma revisão sistemática. Revista de Ciências Médicas e Biológicas 2010;9(2):172-8.

3. Rocha PCX, Moraes CL. Violência familiar contra a criança e perspectivas de intervençáo do Programa Saúde da Família: a experiência do PMF/Niterói (RJ, Brasil). Ciênc Saúde Coletiva 2011;16(7):3285-96.

4. Assis SG, Avanci JQ, Pesce RP, Pires TO, Gomes DL. Notificaçóes de violência doméstica, sexual e outras violências contra crianças no Brasil. Ciênc Saúde Coletiva 2012;17(9):2305-17.

5. Maia AC, Maíra BM. Violência contra crianças e adolescentes no amazonas: análise dos registros. Cad Saúde Pública 2011;27(8):1633-45.

6. Apostólico MR, Nóbrega CR, Guedes RN, Fonseca RMGS, Egry EY. Características da violência contra a criança em uma capital brasileira. Rev Latinoam Enferm 2012;20(2).

7. Silva LMP, Ferriani MGC, Silva MAI. O Poder Judiciário como última instância de proteção às crianças e aos adolescentes: açóes intersetoriais, investimento em recursos humanos e estruturação dos serviços. Rev Latinoam Enferm 2012; 20(3).

8. Silva LMP, Ferriani MGC, Silva MAI. Atuação da Enfermagem frente à violência sexual contra crianças e adolescentes. Rev Bras Enferm 2011;64(5): 919-24.

9. Garbin CAS, Rovida TAS, Joaquim RC, Adrielle MP, Queiroz APDG. Violência denunciada: ocorrências de maus tratos contra crianças e adolescentes registradas em uma unidade policial. Rev Bras Enferm 2011;64(4):665-70.

10. Pfeiffer L, Rosário NA, Cat MNL. Violência contra Crianças e Adolescentes - proposta de classificação dos níveis de gravidade. Revista Brasileira de Educação Médica 2012;36(2):172-9.

11. Koifman L, Menezes RM, Bohrer KR. Abordagem do Tema "Violência contra Crianças" no Curso de Medicina da Universidade Federal Fluminense. Paul Pediatr 2001;29(4):477-82.

12. Silva CJP, Ferreira EF, Paula LPP, Naves MD, Vargas AMD, Zarzar PMPA. A violência urbana contra crian- ças e adolescentes em Belo Horizonte: uma história contada através dos traumas maxilofaciais. Physis Revista de Saúde Coletiva 2001;21(3):1103-20.

13. Zambon MP, Jacintho ACA, Medeiros MM, Guglielminetti R. Violência doméstica contra crianças e adolescentes: um desafio. Rev Assoc Med Bras 2012;58(4):465-71.

14. Algeri S, Souza LM. Violência contra crianças e adolescentes: um desafio no cotidiano da equipe de enfermagem. Rev Latinoam Enferm 2006;14(4).

15. Gonçalves HS, Ferreira AL. A notificação da violência intrafamiliar contra crianças e adolescentes por profissionais de saúde. Cad Saúde Pública 2002;18(1):315-9.

16. Coimbra Junior CEA. Desafios da produçáo e da comunicação científica em saúde no Brasil Cad Saúde Pública 2003;19(1):4-5.

17. Laks J, Werner J, Miranda-Sá Junior L. Psiquiatria forense e Direitos Humanos nos polos da vida: crianças, adolescentes e idosos. Rev Bras Psiquiatr 2006;28 (2):80-5.

18. Lima JA, Alberto MFP. As vivências maternas diante do abuso sexual intrafamiliar. Estudos de Psicologia 2010;15(2):129-36.

19. Santos VA, Costa LF. A violência sexual contra crianças e adolescentes: conhecer a realidade possibilita a ação protetiva. Estudos de Psicologia 2011;28 (4):529-37.

20. Granjeiro IACL. O estudo psicossocial forense como subsídio para a decisão judicial na situação de abuso sexual. Psicol: Teor Pesqui 2008;24(2):161-9.

21. Andrade EM, Nakamura E, Paula CS, Nascimento $\mathrm{R}$, Bordin IA. A visão dos profissionais de saúde em relação à violência doméstica contra crianças e adolescentes: um estudo qualitativo. Saúde Soc São Paulo 2011;20(1):147-55.

22. Baptista RS, França ISX, Costa CMP, Brito RS. Caracterização do abuso sexual em crianças e adolescentes notificado em um programa sentinela. Acta Paul Enferm 2008;21(4):602-8.

23. Cunha JM. A atenção de enfermagem à criança vítima de violência familiar [Tese]. Rio de Janeiro: Instituto Fernandes Figueira; 2007.

24. Fonseca RMGS, Egry EY, Nóbrega CR, Apostólico MR, Oliveira RNG. Reincidência da violência contra crianças no município de Curitiba: um olhar de gênero. Acta Paul Enferm 2012;25(6):895-901.

25. Mascarenhas MDM, Malta DC, Silva MMA, Lima CM, Carvalho MGO, Oliveira VLA. Violência contra a criança: revelando o perfil dos atendimentos em serviços de emergência, Brasil, 2006 e 2007. Cad Saúde Pública 2010;26 (2): 347-57.

26. Guimarães JAT, Villela WV. Características da violência física e sexual contra crianças e adolescentes atendidos no IML de Maceió, Alagoas, Brasil. Cad Saúde Pública 2011;27(8):1647-53.

27. Costa COM, Carvalho RC, Santana MAO, Silva LMS, Silva MR. Avaliação do Programa Nacional de Açôes Integradas e Referenciais (PAIR) para o enfrentamento da violência sexual contra crianças e adolescentes, em Feira de Santana, Bahia. Ciênc Saúde Coletiva 2010;15(2):563-74.

28. Faleiros JM, Matias ASA, Bazon MR. Violência contra crianças na cidade de Ribeirão Preto, São Paulo, Brasil: 
a prevalência dos maus-tratos calculada com base em informaçóes do setor educacional. Cad Saúde Pública 2009;25(2):337-48.

29. Machado HB, Lueneberg CF, Régis EI, Nunes MPP. Abuso sexual: diagnóstico de casos notificados no município de Itajaí/SC, no período de 1999 a 2003, como instrumento para a intervenção com famílias que vivenciam situaçóes de violência. Texto Contexto Enferm 2005; 14:54-63.

30. Brito AMM, Zanetta DMT, Mendonça Rita CV, Barison SZP, Andrade VAG. Violência doméstica contra crianças e adolescentes: estudo de um programa de intervenção. Ciênc Saúde Coletiva 2005;10(1):143-9.

31. Martins CBG, Mello JMHP. Desfecho dos casos de violência contra crianças e adolescentes no poder judiciário. Acta Paul Enferm 2009;22(6):800-7.

32. Melo MC, Cristo RC, Kadama I. Avaliação da assistência multiprofissional dos casos de violência intrafamiliar atendidos em uma unidade de pediatria. Comun Ciênc Saúde 2010;12(3):491-7.

33. Martins CBG, Jorge MHPM. Violência física contra menores de 15 anos: estudo epidemiológico em cidade do sul do Brasil. Rev Bras Epidemiol 2009;12(3):325-37.

34. Cocco M, Silva EB, Jahn AC. Abordagem dos profissionais de saúde em instituiçóes hospitalares a crianças e adolescentes vítimas de violência. Rev Eletr Enf 2010;12(3):491-7.

35. Cocco M, Silva EB, Jahn AC, Poll AS. Violência contra crianças e adolescentes: estratégias de cuidado adotadas por profissionais de saúde. Cienc Cuid Saude 2010;9(2):292-300.

36. Deslandes S, Mendes CHF, Lima JS, Campos DS. Indicadores das açóes municipais para a notificação e o registro de casos de violência intrafamiliar e exploração sexual de crianças e adolescentes. Cad Saúde Pública 2011; 27(8):1633-45 